\title{
RESISTENCIA AL CORTE DE MUROS DE HORMIGON ARMADO
}

\author{
L Gilberto Leiva $\mathrm{H}^{(1)}$ \\ Juan Carlos Montaño E. ${ }^{(2)}$
}

\begin{abstract}
RESUMEN
Se analiza una serie de datos experimentales disponibles en la literatura sobre resistencia al corte de muros de hormigón armado, estudiándose el efecto de los principales parámetros que afectan esta resistencia. Utilizando el modelo que considera por separado las contribuciones $V_{\mathrm{s}}$ del refuerzo transversal y $V_{\mathrm{c}}$ del hormigón, se detectaron dos importantes efectos. En primer lugar, se determinó que el aporte $V_{\mathrm{s}}$ del refuerzo transversal alcanza solamente al 70\% del valor establecido en la norma de diseño del ACI. En segundo lugar, se detectó claramente el efecto de degradación de la contribución del hormigón $V_{\mathrm{c}}$, causada por la demanda de deformaciones no lineales y el consecuente daño asociado. A partir del estudio analítico del mecanismo de transferencia de corte a través de la cabeza de compresión del muro, y del análisis de los resultados experimentales, se ha desarrollado un modelo de resistencia al corte que incluye los efectos antes mencionados. Dados los datos experimentales disponibles, la demanda de deformaciones no lineales en flexión fue evaluada mediante la ductilidad de curvatura alcanzada al instante de la falla. La expresión obtenida mantiene la forma tradicional, incorporando el efecto de la demanda de ductilidad y del nivel de solicitación en flexión sobre la componente $V_{\mathrm{c}}$.
\end{abstract}

\begin{abstract}
By analyzing experimental data available in the literature the importance of the main parameters affecting the shear strength of $\mathrm{R} / \mathrm{C}$ structural walls is studied. Assuming the shear strength as the sum of the strength $V_{\mathrm{c}}$ provided by the concrete shear resisting mechanism and the strength $V_{\mathrm{s}}$ provided by transverse reinforcement, it was found that the $V_{\mathrm{s}}$ strength reaches only $70 \%$ of the value prescribed by the current design codes. Secondly, it was detected the degradation of the concrete shear resisting mechanism due to the non-linear deformation demand and the damage associated. Based on the analysis of the shear transfer mechanism at the wall compression toe, a predictive shear strength model was developed. The concrete mechanism strength reflects the effects of the ductility demand and the flexural loading levels on the critical section.
\end{abstract}

Artículo recibido el 14 de marzo de 2000 y aprobado para su publicación el 26 de febrero del 2001. Se aceptarán comentarios y/o discusiones al artículo hasta cinco meses después de su publicación.

1,2 Departamento de Obras Civiles, Universidad Técnica Federico Santa María, Valparaíso, Chile 


\section{INTRODUCCION}

Las estructuras sufren grandes deformaciones no lineales durante la ocurrencia de un sismo severo. La respuesta presentará características estables y adecuadas en la medida que la estructura tenga la suficiente capacidad de deformarse en el rango no lineal, sin sufrir importante degradación de resistencia, rigidez o capacidad de disipar energía. La filosofía de diseño consiste entonces en asegurar que la fluencia por flexión controle la respuesta, evitando modos de falla frágiles tales como el corte, hasta que una cantidad suficiente de energía se haya disipado por flexión en las secciones críticas del sistema estructural.

Es necesario entonces disponer de herramientas que permitan evaluar tanto la demanda que impondrá el sismo como la capacidad de la estructura. Un diseño por capacidad, a partir de las fuerzas asociadas a la demanda de deformaciones, parece ser adecuado. Sin embargo, de acuerdo a las características de la demanda, el daño se acumula en las estructuras, degradándose su capacidad. El objetivo de este trabajo es evaluar, a partir de datos experimentales disponibles en la literatura, el efecto de la degradación de resistencia al corte en muros de hormigón armado, sometidos a altos niveles de deformaciones no lineales en flexión. Los resultados permiten desarrollar las bases de un modelo de resistencia al corte que incluye dicho efecto.

\section{EVALUACION DE MODELOS DE RESISTENCIA AL CORTE}

Se realizó una recopilación y estudio de la información disponible en la literatura, tanto analítica como experimental, sobre el comportamiento al corte de muros de hormigón armado. De la información experimental, y con el objeto de eliminar la influencia de los parámetros relacionados con los elementos de borde, se seleccionaron los datos de muros de sección rectangular que desarrollaron modos de falla por corte, correspondientes a las siguientes investigaciones y reportes: PCA (Cárdenas et.al., 1973); Universidad Católica de Chile (Jordan y Lüders 1993; Larenas, 1994; Kou, 1995; Hidalgo y Jordan, 1996); Wood, 1989; Pilakoutas y Elnashai, 1995; Pilakoutas y Elnashai, 1995 y UNAM (Hernández y Zermeño, 1980). Se trata de un total de 43 especímenes, de espesor entre 6 y $12 \mathrm{~cm}$ y esbeltez $M / V \ell_{w}$ entre 0.35 y 3.36 , donde $M$ y $V$ son el momento flector y la fuerza de corte en la base del muro y $\ell_{w}$ es la longitud de la sección transversal. La cuantía de refuerzo longitudinal varía entre 0.0027 y 0.0302 . La cuantía de refuerzo transversal varía entre 0.0 y 0.010 .

Los modelos existentes de resistencia al corte han sido derivados tanto de resultados experimentales como a partir de conceptos básicos de la mecánica estructural. La mayoría de las provisiones de diseño, incluyendo las del ACI 318, siguen el primero de estos enfoques. La 
información recopilada incluye las ecuaciones de diseño del ACI 318 (ACI, 1995) y japonesas (AIJ, 1988) y los modelos presentados en los siguientes reportes: Barda et.al., 1977; Hirosawa et.al., 1988 y Hernández y Zermeño, 1980. Como una primera forma de ilustrar las diferencias entre los modelos, se calculó la resistencia al corte de un muro de características similares a las del espécimen SW-8 del programa experimental de la P.C.A. (Cárdenas et.al., 1973), con cada uno de los modelos estudiados. El espécimen corresponde a un elemento de sección transversal de $7.6 \mathrm{~cm}$ de espesor $b$ y $190.5 \mathrm{~cm}$ de longitud $\ell_{w}$, con cuantías de refuerzo longitudinal y transversal de 0.0289 y 0.0027 respectivamente. Los resultados se presentan en la Fig. 1, en términos del esfuerzo resistente de corte medio $v_{\mathrm{n}}$ sobre la sección efectiva $b d$ del muro, en función de la esbeltez $M / V \ell_{w}$. Los círculos negros en cada curva indican el rango de validez de los modelos de origen empírico, definido por los datos experimentales utilizados en la calibración de cada modelo. El cuadrado negro representa la resistencia experimental del espécimen con esbeltez igual a 1.0. Se puede apreciar una gran dispersión entre los resultados, especialmente en el rango de valores bajos de esbeltez.

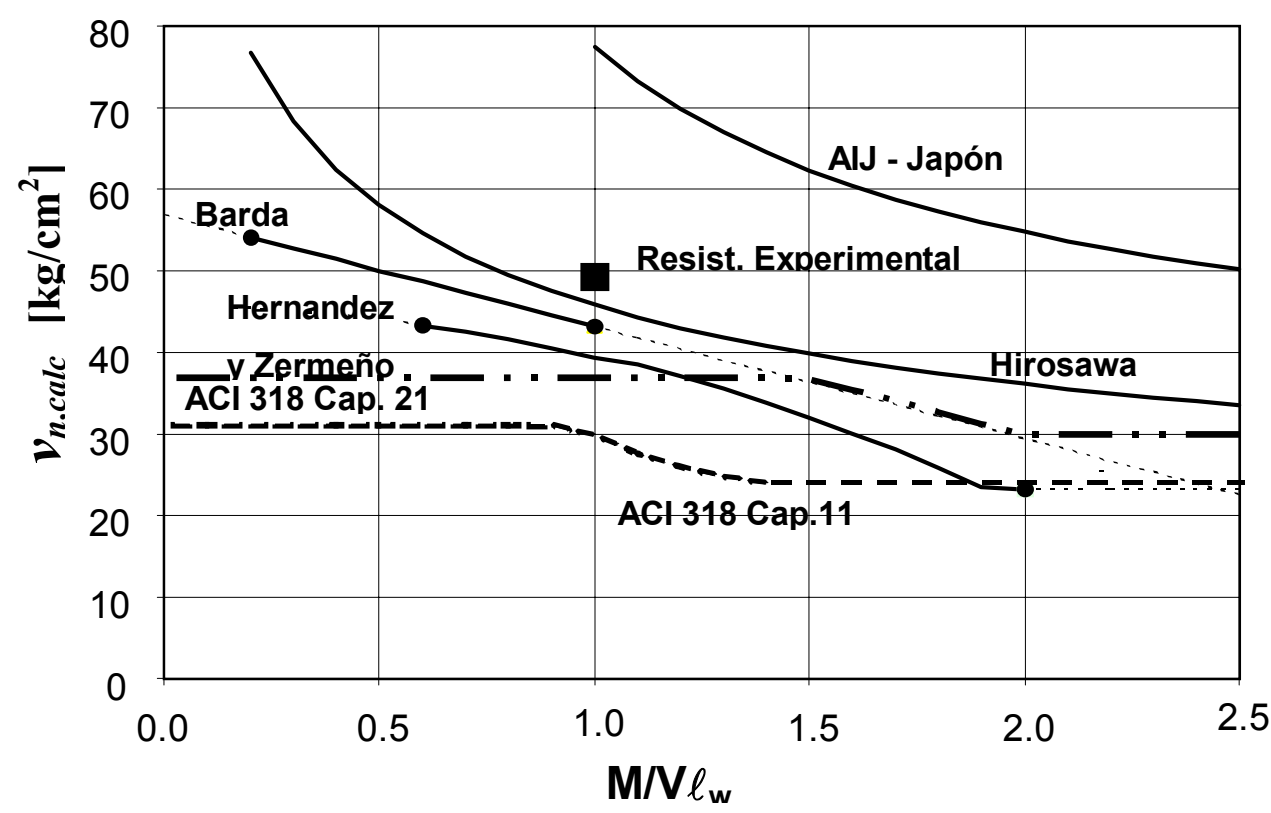

Fig. 1 Resistencia al corte de un muro similar al espécimen SW-8 (Cárdenas, 1973), calculada según diversos modelos 
Utilizando los datos experimentales disponibles, los modelos analíticos recopilados fueron estudiados comparando los valores de la resistencia al corte calculada y la medida. Los resultados, en términos del cuociente entre ambos valores, se resumen en la Tabla 1. Para cada modelo se indica el valor medio, el coeficiente de variación (desviación estándar normalizada con respecto al valor medio) y los valores con probabilidad $90 \%$ y $10 \%$ de ser excedidos, $P 90$ y $P 10$ respectivamente, calculados suponiendo que los resultados obtenidos presentan una distribución de tipo normal. El análisis detallado de estos resultados permite verificar las hipótesis e identificar los parámetros más relevantes que afectan la resistencia al corte, así como las bondades de cada modelo. Como ejemplo, en las Figs. 2a y $2 \mathrm{~b}$ se presentan las distribuciones de los valores del cuociente entre las resistencias calculada y experimental para la ecuación de diseño del Capítulo 21 del ACI-318, en función de la esbeltez $M / V \ell_{w}$ del muro y de la cuantía de refuerzo transversal $\rho_{\mathrm{h}}$ respectivamente.

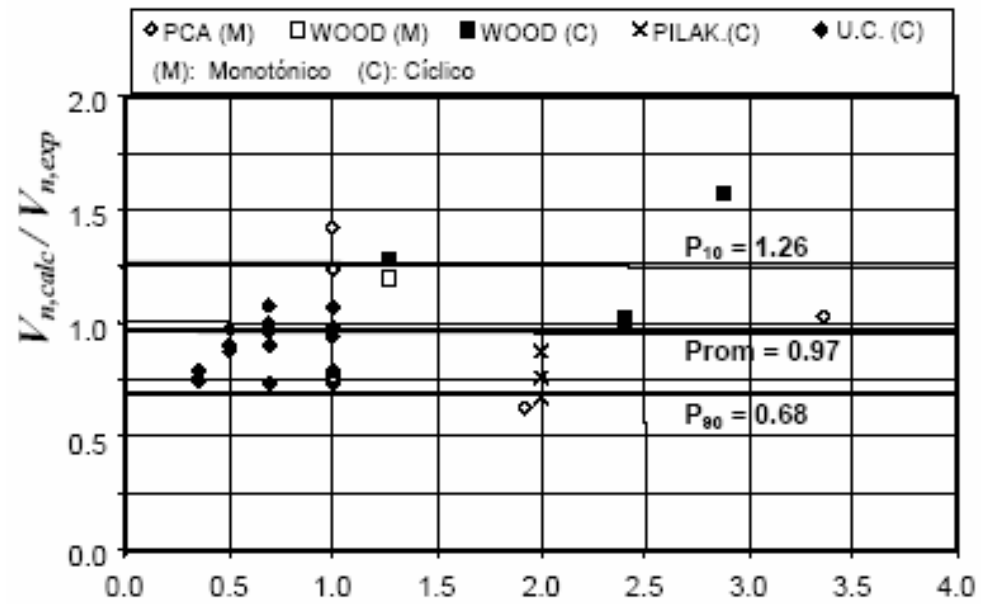

(a) Esbeltez $\mathbf{M} / \mathbf{V} \ell_{w}$

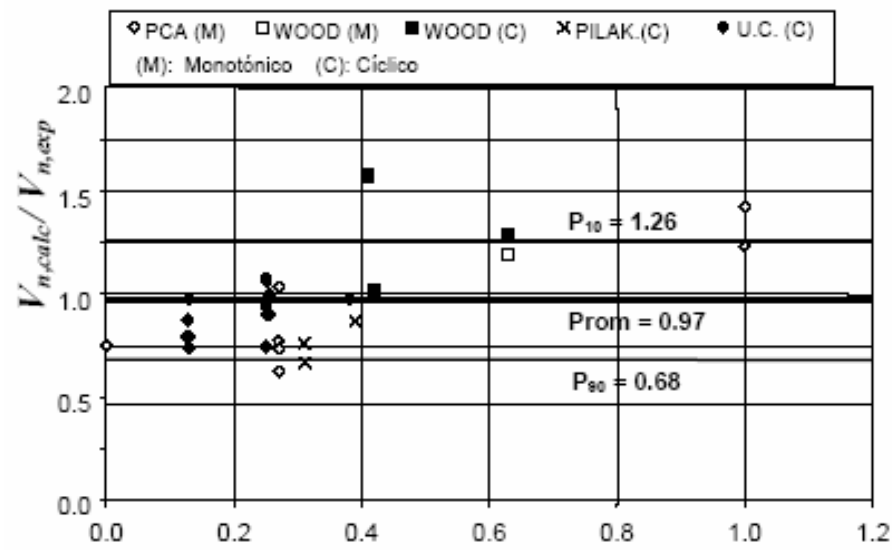

(b) Cuantía de refuerzo Transversal $\rho_{\mathrm{h}} \%$

Fig. 2 Cuociente $V_{n, \text { calc }} / V_{n, \text { exp }}$ para ecuación de diseño Norma ACI 318-Cap. 21 
Se observa que la resistencia calculada es relativamente consistente con los valores experimentales para muros con esbeltez baja, $M / V \ell_{w}<1$. Sin embargo, para muros más esbeltos, en los que predomina el comportamiento por flexión, el modelo tiende a sobrestimar la resistencia y mostrar una alta dispersión. Con respecto a la cuantía de refuerzo transversal, se observa claramente que las resistencias calculadas con el ACI 318 tienden a ser muy poco conservadoras para valores de $\rho_{\mathrm{h}}$ mayores que $0.40 \%$, observación similar a los resultados descritos por Wood (1990). Resultados similares obtenidos con otros modelos indican la necesidad de revisar las hipótesis básicas de dichos modelos.

\section{BASES PARA EL DESARROLLO DE UN MODELO DE RESISTENCIA AL CORTE}

\section{Modelo Conceptual}

Los resultados analizados han permitido identificar los principales aspectos a considerar en el desarrollo de un modelo de resistencia al corte para muros de hormigón armado: a) se debe revisar la hipótesis generalmente aceptada que todo el refuerzo transversal que cruza una grieta diagonal alcanza la fluencia; y b) se debe incorporar el efecto de degradación de la resistencia al corte en elementos que están sometidos a demandas de deformación no lineal en flexión. El modelo conceptual propuesto originalmente para columnas (ATC, 1981), que aparece en la Fig. 3 , es adecuado a estos fines. Se supone que la degradación de la resistencia al corte $V_{\mathrm{n}}$ ocurre a partir de un cierto límite, hasta que la resistencia alcanza un valor residual, como se muestra en la

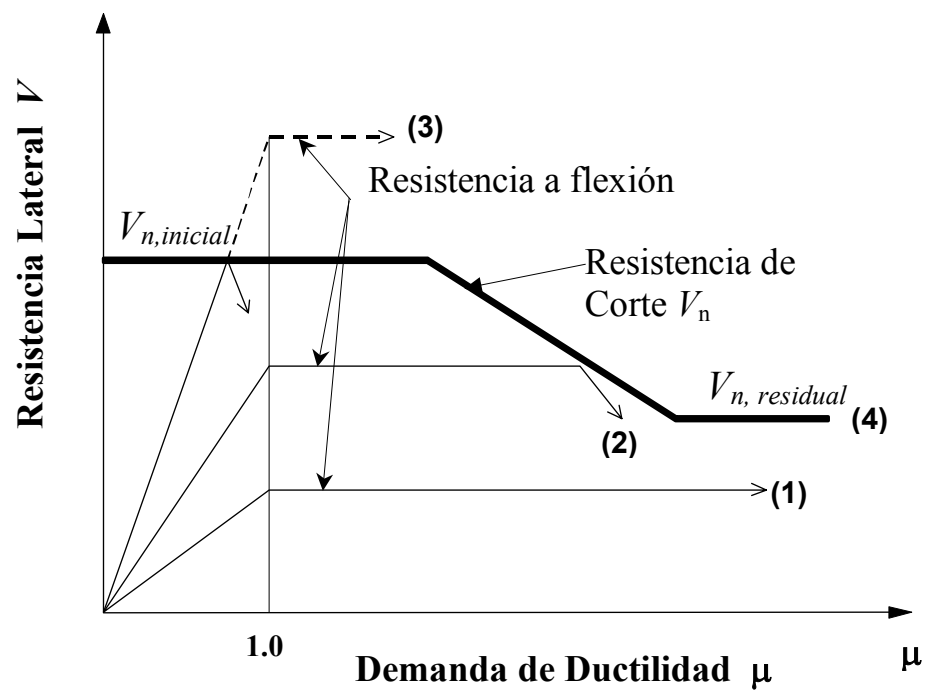

Fig. 3. Modelo conceptual de resistencia al corte (ATC, 1981) 
curva (4) de la figura. Dependiendo de los valores relativos entre la resistencia al corte y la resistencia a flexión, un elemento puede desarrollar uno de los tres tipos de comportamiento que se muestran en la Fig. 3. En el primer caso, curva (1), la resistencia a la flexión es menor que la resistencia residual de corte, pudiendo el elemento desarrollar una falla dúctil por flexión, después de alcanzar altos niveles de deformaciones no lineales. En el caso (2), el elemento posee una resistencia inicial al corte suficiente para desarrollar la fluencia en flexión. Sin embargo, después de haber desarrollado un cierto nivel de ductilidad, ocurre la falla por corte debido a que la capacidad de corte se degrada más rápidamente que la de flexión. En el caso (3), la resistencia a la flexión es mayor que la resistencia inicial al corte, produciéndose una falla frágil por corte. La resistencia al corte $V_{\mathrm{n}}$, en la etapa posterior al agrietamiento diagonal, se expresa en la forma tradicional como la superposición de las contribuciones del hormigón $V_{\mathrm{c}}$ y la del refuerzo transversal $V_{\mathrm{s}}$. Se supone que la degradación afecta solamente a $V_{\mathrm{c}}$.

\section{Contribución del Refuerzo Transversal $V_{\mathrm{s}}$}

La contribución del refuerzo $V_{\mathrm{s}}$ ha sido calculada tradicionalmente considerando que todo el refuerzo transversal que cruza a través de una grieta diagonal a $45^{\circ}$ alcanza la fluencia. Sin embargo, evidencia experimental muestra que el ancho de la grieta diagonal es mínimo en las zonas cercanas a los extremos de las secciones del muro. En estas condiciones, no es posible que el refuerzo transversal que cruza la grieta en estas zonas extremas desarrolle la fluencia. Como consecuencia de este y otros factores, como la inclinación de la grieta, los valores de la contribución del refuerzo transversal $V_{\mathrm{s}}$ calculados con las expresiones tradicionales son mayores que los reales.

Con el objeto de determinar el valor de $V_{\mathrm{s}}$ a partir de los datos experimentales, es útil expresar esta contribución como una fracción $\alpha$ del $V_{\mathrm{s}}$ tradicional:

$$
V_{s}=\alpha\left(\frac{A_{s h}}{s b} f_{y} b d\right)=\alpha\left(\rho_{h} f_{y} b d\right)
$$

donde $A_{\text {sh }}$ es el área del refuerzo transversal a distancia $s ; f_{y}$ es la tensión de fluencia del refuerzo transversal; $b$ y $d$ son el espesor y la longitud efectiva de la sección respectivamente; $\rho_{\mathrm{h}}$ es la cuantía de refuerzo transversal $\left(\rho_{\mathrm{h}}=A_{\mathrm{sh}} / b s\right)$. El factor $\alpha$ se puede interpretar como la efectividad del refuerzo transversal. En la Fig. 4 se han graficado los valores experimentales de la resistencia $v_{\mathrm{n}}=V_{\mathrm{n}} / b d$ en función de $\rho_{\mathrm{n}} f_{\mathrm{y}}$. Los datos se presentan ordenados en tres grupos según la esbeltez $M / V \ell_{w}$ de los muros. El análisis de los datos experimentales ha mostrado que la relación entre la resistencia al corte y la cuantía de refuerzo transversal mantiene una tendencia similar, cualquiera que sea el parámetro (esbeltez, fuerza axial, etc.) utilizado para ordenar los 
datos:. En la misma figura se muestra la recta ajustada a la totalidad de los datos mediante una regresión lineal. La recta tiene una pendiente igual a 0.7 , valor que corresponde a la efectividad $\alpha$ del refuerzo transversal.

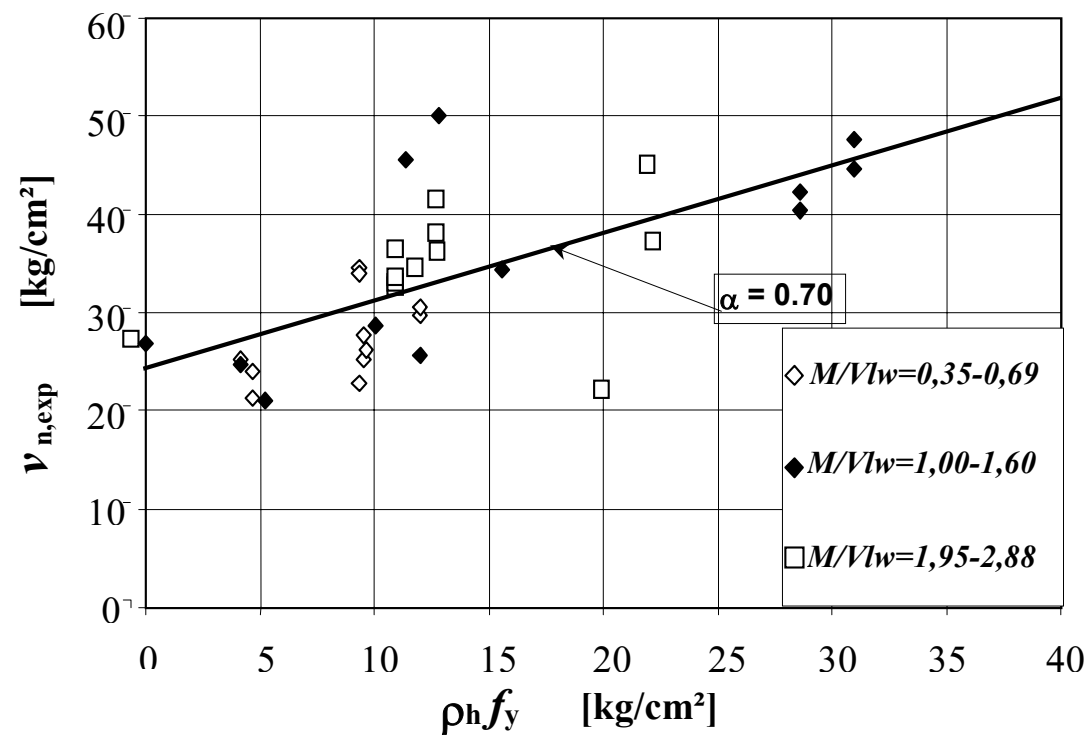

Fig. 4. Resistencia experimental al corte $\mathrm{v} / \mathrm{s}$ resistencia del refuerzotransversal

\section{Contribución del Hormigón $V_{\mathrm{c}}$}

A efectos de estudiar la influencia de los diversos parámetros que inciden en la contribución del hormigón a la resistencia al corte, se calculó el valor de $V_{\mathrm{c}}$ como la diferencia entre el $V_{\mathrm{n}}$ medido y $V_{\mathrm{s}}$ calculado según la Ec. 1, con $\alpha$ igual a 0.7. En las Figs. 5(a) y 5(b) se han graficado los

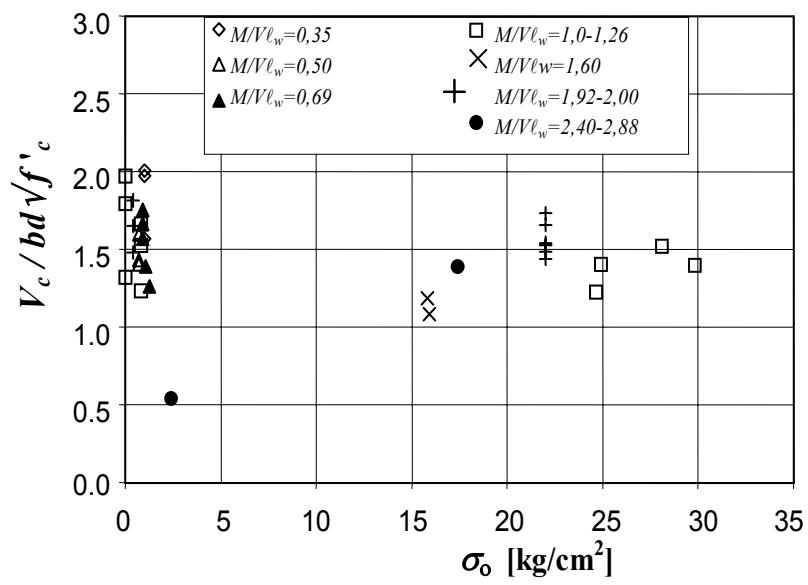

(a)

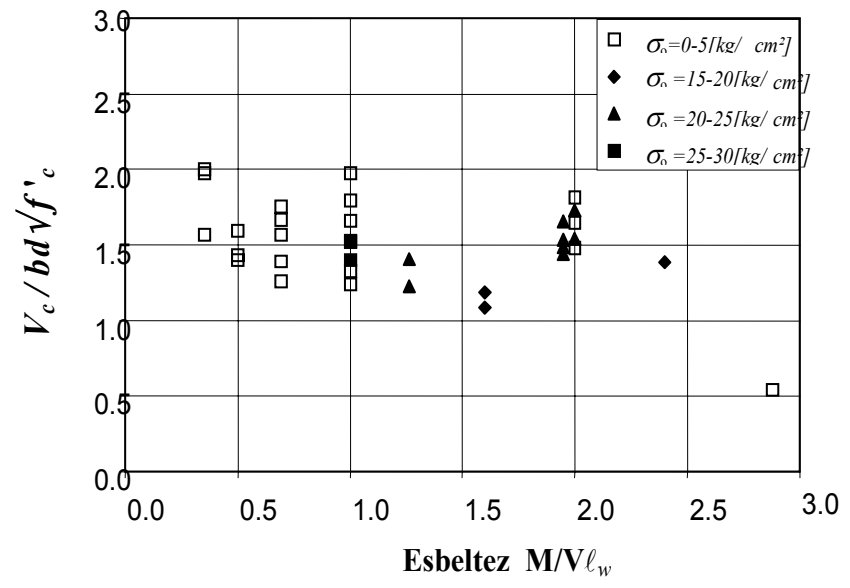

( b)

Fig. 5 Valores experimentales de $V_{\mathrm{c}}$ en función del esfuerzo axial medio $\sigma_{\mathrm{o}} \mathrm{y}$ de la esbeltez

$$
M / \ell_{\mathrm{w}}
$$


valores experimentales de $V_{\mathrm{c}}$ en función de la esbeltez $M / V l_{\mathrm{w}}$ y del esfuerzo axial medio $\sigma_{\mathrm{o}}$. Tal como se aprecia en los gráficos, no existe una tendencia definida en los resultados.

La ductilidad alcanzada al instante de la falla fue calculada utilizando los datos disponibles. Conocido el valor del momento flector $M$ en la sección más solicitada al instante de la falla por corte, mediante un análisis seccional basado en la hipótesis de secciones planas y las leyes de comportamiento de los materiales, se determinó la profundidad de la línea neutra $\mathrm{kd}$, la curvatura $\phi$ y la ductilidad de curvatura $\mu_{\phi}$. Dado que no se disponía de otros datos, se decidió utilizar esta ductilidad como parámetro para evaluar la demanda de deformaciones no lineales sobre los muros. Los resultados aparecen en la Fig. 6, en términos de los valores experimentales de $V_{\mathrm{c}}$ en función de la ductilidad de curvatura desarrollada al instante de la falla por corte. A los muros que no alcanzaron la curvatura de fluencia se les ha asignado ductilidad nula. Se aprecia claramente que solamente los muros de esbeltez igual o superior a uno desarrollaron cierto nivel de ductilidad. De igual forma se aprecia que existe una tendencia a la disminución de la resistencia con el aumento de la demanda de ductilidad.

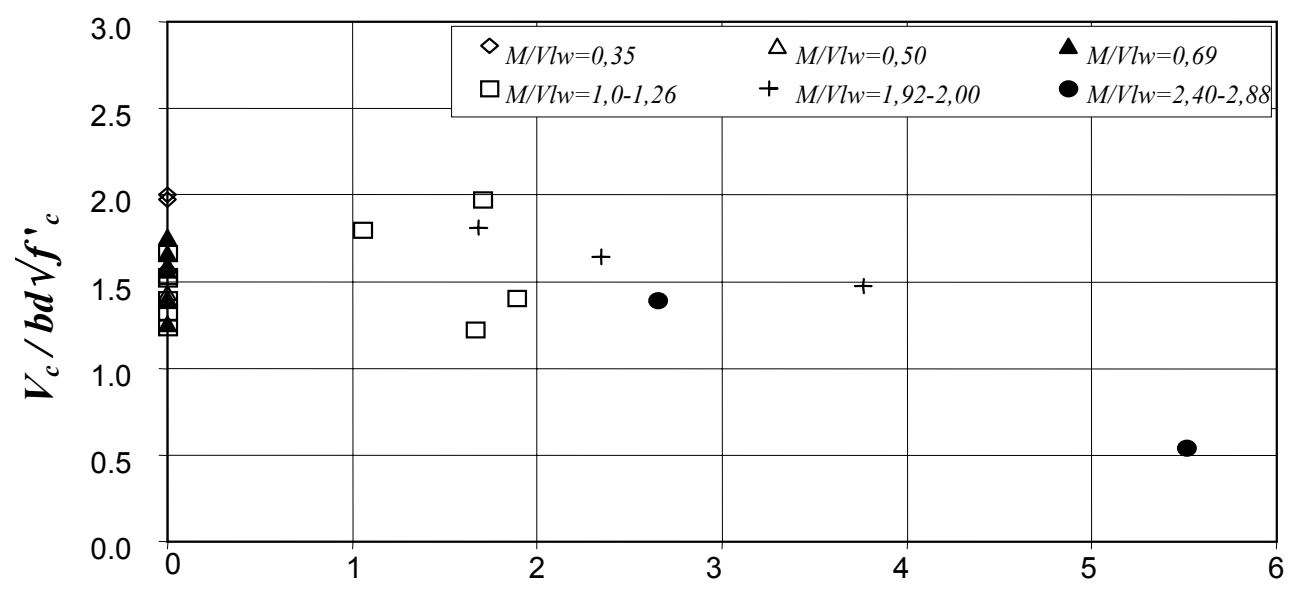

Fig. 6 Influencia de la ductilidad de curvatura $\mu^{\phi}$ sobre la resistencia Vc. Muros agrupados según esbeltez $\mathrm{M} / \mathrm{V} \ell_{\mathrm{w}}$ 


\section{MODELO DE RESISTENCIA AL CORTE}

Los resultados anteriores han permitido detectar el efecto de la demanda de ductilidad sobre la resistencia al corte. Con el objeto de estudiar posibles correlaciones entre la demanda de ductilidad y otros parámetros que afectan la resistencia, se realizó un estudio analítico del mecanismo resistente de los muros. La contribución del hormigón $V_{\mathrm{c}}$ incluye mecanismos tales como la transmisión de corte a través de la cabeza de compresión del muro, el corte transmitido por medio de la trabazón de los agregados, el efecto tarugo (dowel action) del refuerzo longitudinal y el efecto del esfuerzo axial. El análisis que se presenta a continuación está basado en el mecanismo de transferencia de corte a través de la cabeza de compresión. En la Fig. 7 se muestra las fuerzas en el muro sometido a un corte $V$ y un esfuerzo axial $\sigma_{0}$. Las fuerzas resultantes en la cabeza de compresión, de longitud $k d$, son la compresión $C_{\mathrm{u}}$ y el corte $V_{\mathrm{c}}$. El esfuerzo de compresión medio $n$ en esta zona es:

$$
n=\frac{C_{u}}{b(k d)}
$$

Suponiendo comportamiento muy cercano al elástico en la cabeza de compresión, el esfuerzo máximo de corte $v_{\max }$ en este lugar es:

$$
v_{\max }=\frac{3 V}{2 b(k d)}
$$

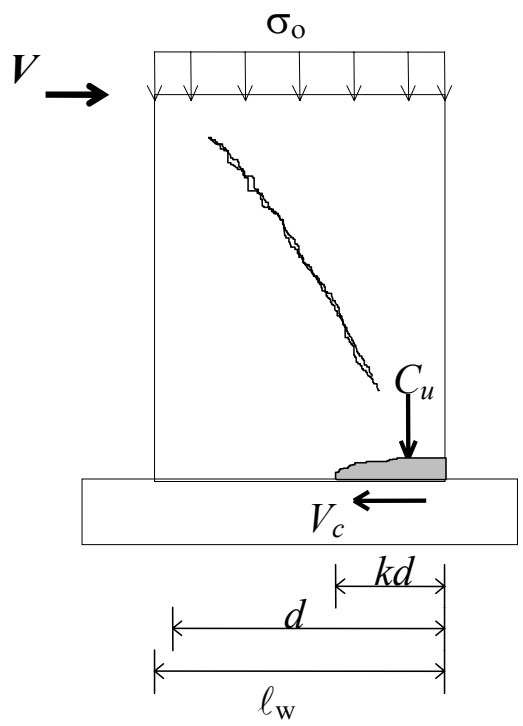

Figura 7 Fuerzas en el Muro

Mediante las ecuaciones de Mohr se puede calcular el esfuerzo principal de tracción en función de los esfuerzos axial $n$ y de corte $v_{\max }$. Se supone que el evento que define el valor de la 
contribución $V_{\mathrm{c}}$ corresponde al instante en que el esfuerzo principal de tracción alcanza la resistencia del hormigón $f_{t}=\beta \sqrt{f^{\prime}{ }_{c}}$. Se despeja entonces $v_{\max }$, y reemplazando según las Ecs. 2 y 3 , se obtiene la siguiente expresión para $V_{\mathrm{c}}$ :

$$
\frac{V_{c}}{b d}=\frac{2}{3} \beta \sqrt{f_{c}^{\prime}}\left(\frac{k d}{d}\right) \sqrt{1+\left(\frac{1}{\beta}\right) \frac{C_{u}}{b(k d) \sqrt{f^{\prime}{ }_{c}}}}
$$

Con el propósito de analizar esta expresión, los términos que aparecen en la Ec. (4) fueron calculados para cada uno de los datos experimentales utilizados en este estudio. La profundidad de la línea neutra $k d$ y la resultante de compresiones $C_{\mathrm{u}}$ se calcularon con el valor del momento flector $M$ y del esfuerzo axial medio $\sigma_{\mathrm{o}}$ en la sección crítica al instante de la falla por corte. De igual forma se calculó la curvatura $\phi$ y la ductilidad de curvatura $\mu_{\phi}$ desarrolladas. El momento resistente $M_{\mathrm{n}}$ de la sección crítica fue calculado considerando como criterio de falla por flexión una deformación máxima del hormigón de 0.003 .

En primer lugar se analizó la relación entre el término $k d / d$ y la ductilidad de curvatura desarrollada. Los valores de ambos parámetros han sido graficados en la Fig. 8, donde se aprecia que existe una clara correlación entre ellos, la que puede ser representada por la siguiente función:

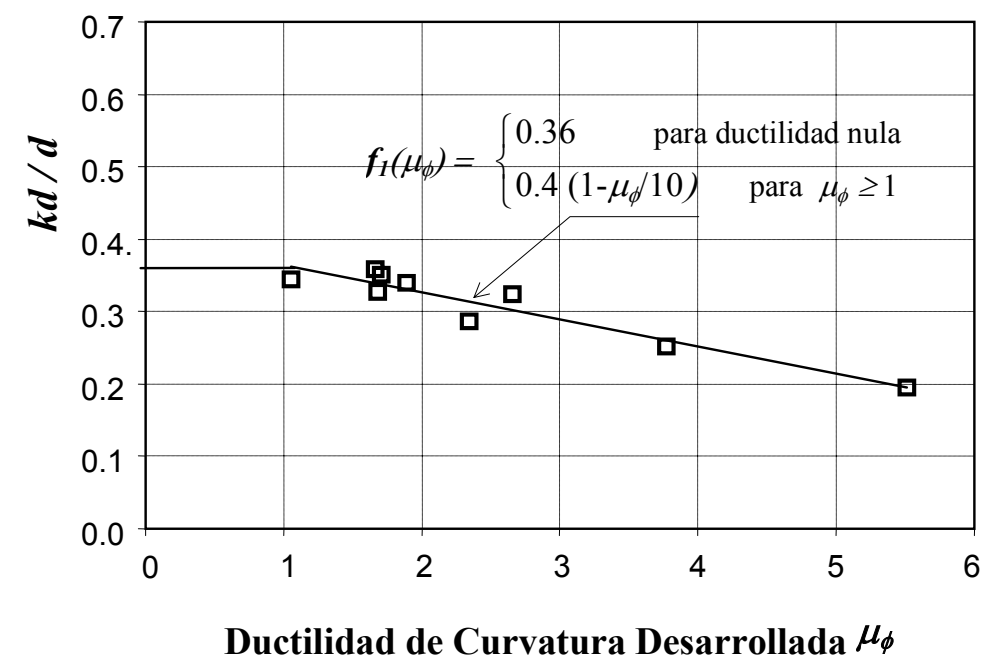

Fig. 8 Cuociente $(k d / d)$ en función de la ductilidad de curvatura desarrollada $\mu_{\phi}$. 


$$
\left(\frac{k d}{d}\right)=f_{1}\left(\mu_{\phi}\right)=\left\{\begin{array}{lc}
0.36 & \text { sin requerimiento de ductilidad } \\
0.40\left(1-\mu_{\phi} / 10\right) & \text { para } \mu_{\phi} \geq 1
\end{array}\right.
$$

La resultante de compresiones $C_{\mathrm{u}}$ depende del nivel de fuerza axial y momento flector en la sección crítica del muro, de las dimensiones de la sección y del brazo entre las resultantes de tracción y compresión. Se analizó la relación existente entre el término de la raíz de la Ec. 4, calculado a partir de los datos experimentales, y los parámetros antes señalados. Se decidió utilizar el cuociente $\left(M / M_{n}\right)$ como índice del nivel de solicitación en flexión. Entre varias alternativas consideradas, la que se presenta en la Fig. 9 fue la que entregó la mejor correlación, obteniéndose una relación del tipo:

$$
1+\left(\frac{1}{\beta}\right) \frac{C_{\text {ukd }} \sqrt{f_{c}^{\prime}}}{{ }^{\prime}}=g\left(\frac{M}{M_{n}} \rho_{\text {flex }}\right)=A+B\left(\frac{M_{n}}{M_{n}} \rho_{\text {flex }}\right)
$$

Donde $\rho_{\text {flex }}$ es la cuantía total de refuerzo longitudinal y $A$ y $B$ son constantes. La función obtenida evalúa los efectos del nivel de solicitación en flexión y del refuerzo longitudinal sobre la resistencia $V_{\mathrm{c}}$. Este resultado coincide con observaciones experimentales que han mostrado que la resistencia al corte tiende a aumentar con el incremento de la cuantía de refuerzo longitudinal

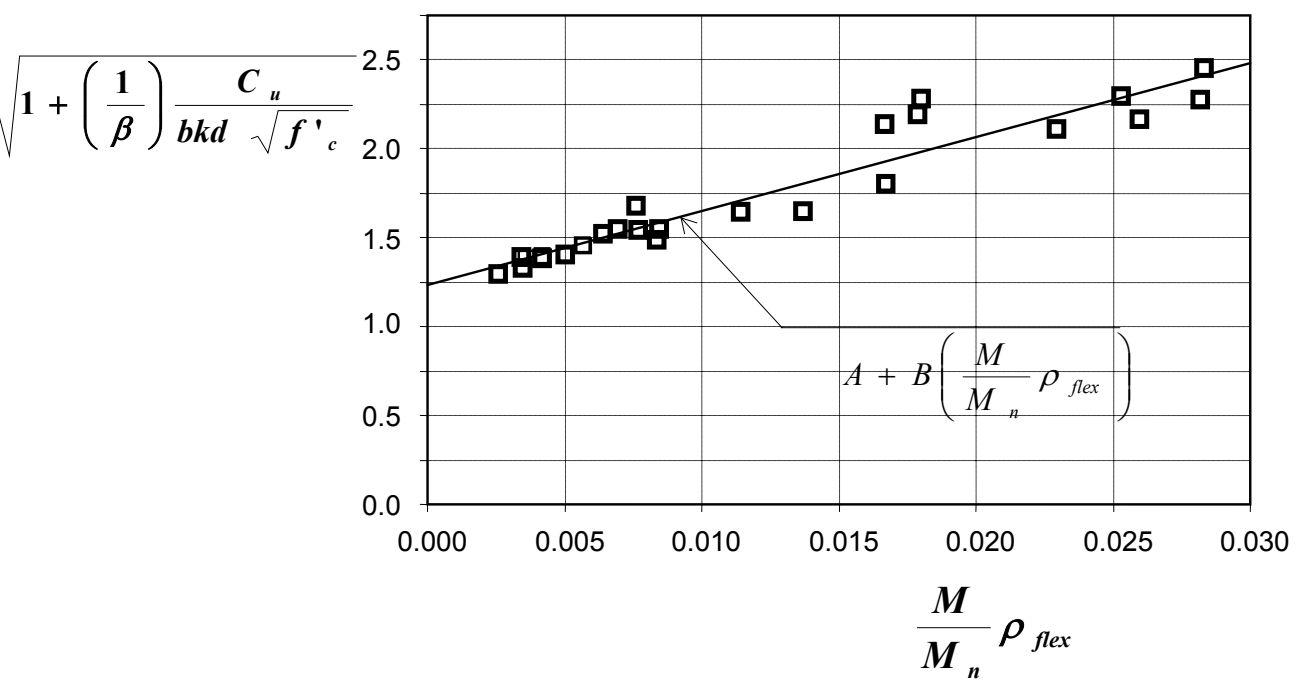

Fig. 9 Resultante de compresiones $C_{u}$ en la cabeza del muro en función de la razón de momentos y la cuantía de refuerzo longitudinal $\left(M / M_{n}\right) \rho_{\text {flex }}$. 
de los elementos de borde y del alma (Wood, 1990). También se ha encontrado que el refuerzo longitudinal es efectivo como refuerzo de corte en muros con esbeltez baja, además de ser efectivo para producir una mejor distribución de las grietas y reducir su espesor (Barda et.al., 1977). En esta relación no aparece el esfuerzo axial $\sigma_{0}$ sobre el muro, ya que el efecto de este parámetro no es significativo en el rango de valores considerados en este trabajo. De esta forma, combinando las ecuaciones 4,5 y 6 , se puede escribir:

$\frac{V_{c}}{b d \sqrt{f^{\prime}{ }_{c}}}=C \cdot g\left[\left(\frac{M}{M_{n}} \cdot \rho_{f l e x}\right)\right] \cdot f\left(\mu_{\phi}\right)$

Donde $f$ y $g$ son las funciones obtenidas del análisis estadístico de los datos experimentales. La resistencia, normalizada con respecto a las funciones $f$ y $g$, fue analizada con respecto al esfuerzo axial y a la esbeltez del muro. Se verificó que los datos disponibles no son suficientes para evaluar el efecto del esfuerzo axial. Con respecto a la esbeltez, en la Fig. 10 se aprecia que existe una cierta tendencia, la que ha sido representada por la función $h$, que aparece en la misma figura.

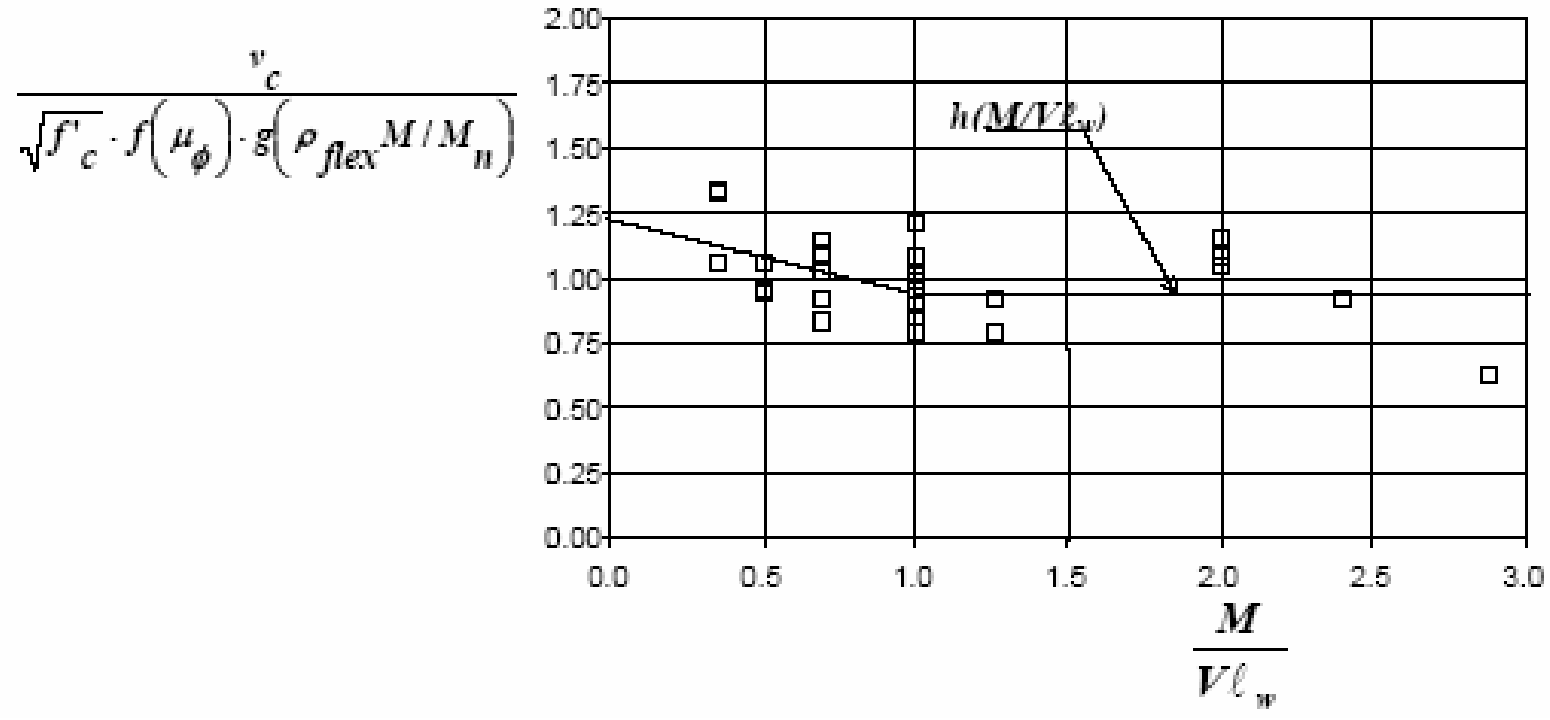

Fig. 10 Contribución del hormigón a la resistencia al corte en función de la esbeltez $M N \ell_{w}$ 
Finalmente, después de ajustar las constantes presentes en las expresiones obtenidas, se obtiene la siguiente ecuación para evaluar la resistencia al corte de muros de hormigón armado, en unidades $\mathrm{Kg} / \mathrm{cm}^{2}$ :

$$
V_{n}=V_{c}+V_{s}=2.0 \cdot\left[1+10 \frac{M}{M_{n}} \cdot \rho_{f l e x}\right] \cdot H\left(\frac{M}{V \ell_{\omega}}\right) \cdot f\left(\mu_{\phi}\right) \cdot \sqrt{f_{c}^{\prime} \cdot} \cdot b d+\alpha\left(\frac{A_{s h}}{s b} f_{y} b d\right)
$$

donde:

$$
\begin{aligned}
& H\left(\frac{M}{V \ell_{\omega}}\right)=\left\{\begin{array}{lr}
1-0.25 \frac{M}{V \ell_{\omega}} & \text { para }\left(M / V \ell_{\omega}\right) \leq 1 \\
0.75 & \text { para }\left(M / V \ell_{\omega}\right)>1
\end{array}\right. \\
& f\left(\mu_{\phi}\right)=\left\{\begin{array}{lc}
0.90 & \text { sin demanda de ductilidad } \\
1-0.10 \mu_{\phi} & \text { para } \mu_{\phi} \geq 1
\end{array}\right. \\
& \alpha=0.70
\end{aligned}
$$

\section{DISCUSION DE RESULTADOS}

El modelo conceptual presentado es válido para elementos que desarrollan deformaciones no lineales y alcanzan la fluencia en flexión, que es el comportamiento deseado de acuerdo a la filosofía de diseño sismorresistente. No es aplicable entonces a muros bajos en los que predomina el corte. Los valores de la esbeltez, cuantías y otros parámetros que definen el límite entre ambos tipos de comportamiento deberían ser materia de estudios posteriores.

La expresión obtenida para evaluar la resistencia al corte mantiene la forma tradicional, incorporando el efecto de la demanda de ductilidad y del nivel de solicitación en flexión sobre la componente $V_{\mathrm{c}}$. Los términos que contienen la ductilidad alcanzada $\mu_{\phi} \mathrm{y}$ el cuociente $M / M_{\mathrm{n}}$ de alguna manera evalúan el nivel de daño por flexión en el elemento al momento de la falla por corte. La importancia relativa de estos factores depende del nivel de deformaciones. Previo a la fluencia, $M / M_{\mathrm{n}}$ crece mientras $\mu_{\phi}$ permanece constante igual a cero. Al llegar y sobrepasar el nivel de fluencia, $M / M_{\mathrm{n}}$ permanecerá en un valor muy cercano a uno, mientras $\mu_{\phi}$ crece. Los términos que incluyen la esbeltez y la ductilidad aparecen separados. Sin embargo, como muestran los datos experimentales, existe una relación bastante directa entre ambos. De hecho, el 
nivel de deformaciones no lineales en flexión que el muro es capaz de alcanzar depende directamente de su esbeltez.

La expresión presentada incluye una contribución $V_{\mathrm{s}}$ del refuerzo transversal menor que la tradicionalmente especificada en las normas de diseño. Con el objeto de verificar el valor obtenido de la efectividad $\alpha$ del refuerzo transversal, se repitió el procedimiento de obtención del modelo para diferentes valores de $\alpha$. Para cada uno de los muros se calculó la resistencia predicha con cada uno de los nuevos modelos, así como los cuocientes entre las resistencias predichas y la experimental. Para cada grupo de resultados, correspondiente a un valor dado de $\alpha$, se calculó el coeficiente de variación, graficándose los resultados en la Fig. 11. Tal como se aprecia en el gráfico, el valor inicialmente considerado de $\alpha=0.70$ está muy cerca del punto de mínimo coeficiente de variación, siendo adecuado para representar la efectividad del refuerzo transversal.

Dados los datos experimentales disponibles, con un valor máximo de la demanda de ductilidad $\mu_{\phi}$ cercano a seis, el modelo presentado no permite evaluar la resistencia de muros con demandas mayores. De la misma manera, no ha sido posible evaluar el valor límite de la ductilidad de curvatura a partir de la cual no existe degradación y la resistencia se mantiene constante a un valor residual. Con respecto a la esbeltez, los datos experimentales incluyen un valor máximo cercano a tres. Sin embargo, el modelo debería ser válido para muros más esbeltos, ya que para esbelteces mayores que uno la influencia de este parámetro permanece constante.

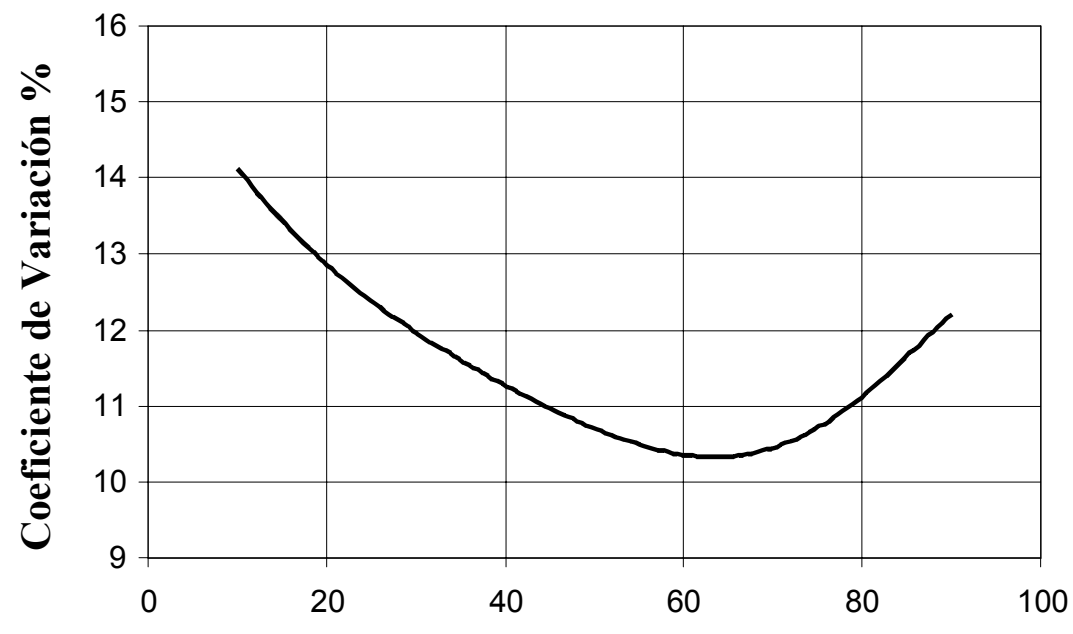

Efectividad $\alpha$ del Refuerzo Transversal \%

Figura 11 Coeficiente de variación de la distribución de los cuocientes entre la resistencia calculada con modelo propuesto para $\alpha^{\phi}$ dado y la experimental, en función de la efectividad $\alpha$ del refuerzo transversal 
Con el objeto de evaluar la expresión desarrollada, para cada uno de los datos disponibles de muros que fallan por corte, se calculó el cuociente entre la resistencia calculada con el modelo propuesto y el valor experimental. Los resultados, en función de la esbeltez, se presentan en la Fig. 12. El valor medio de la distribución es 1.0 y el coeficiente de variación es del 10\%. Suponiendo que los valores calculados son una distribución normal, los valores con probabilidad $10 \%$ y $90 \%$ de ser excedidos son 1.14 y 0.87 respectivamente.

Finalmente, se utilizó el modelo para estudiar casos de muros que presentan una resistencia al corte suficiente para permitir que ocurra falla por flexión u otro modo diferente al corte. El primer caso corresponde a elementos diseñados de acuerdo a la filosofía actual de diseño sísmico. Para estos elementos, el término $M / M_{\mathrm{n}}$ es constante e igual a uno, lo cual permite simplificar la expresión del modelo propuesto. Utilizando datos disponibles en los mismos reportes antes mencionados, se calcularon los valores del cuociente entre la resistencia determinada con el modelo propuesto y la demanda de corte $V_{\mathrm{d}}$ al instante de la falla. Los resultados se presentan en la Fig. 13, en función de la demanda de ductilidad de curvatura. Tal como se esperaba, prácticamente todos los valores son mayores que la unidad, indicando que la resistencia al corte es mayor que la fuerza de corte medida al instante de la falla. El único caso que muestra un valor menor a uno corresponde a un espécimen que falló por flexión-corte. Estos resultados confirman la validez del modelo desarrollado.

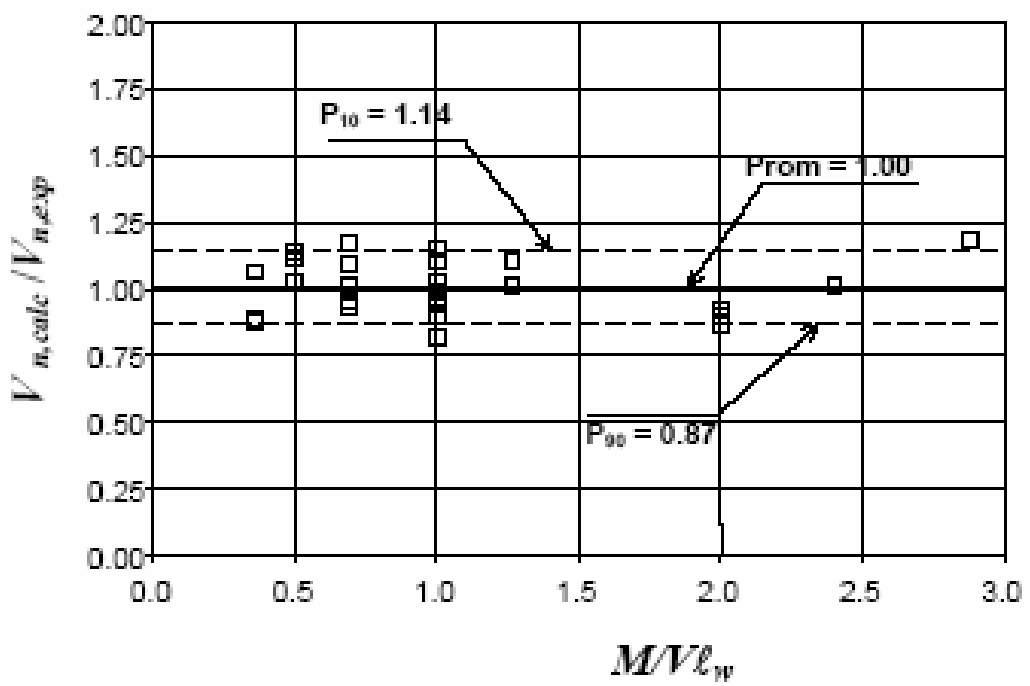

Fig. 12 Cuociente de resistencias calculadas con el modelo propuesto y experimentales, en función de la esbeltez $M N \ell_{\mathrm{w}}$. 


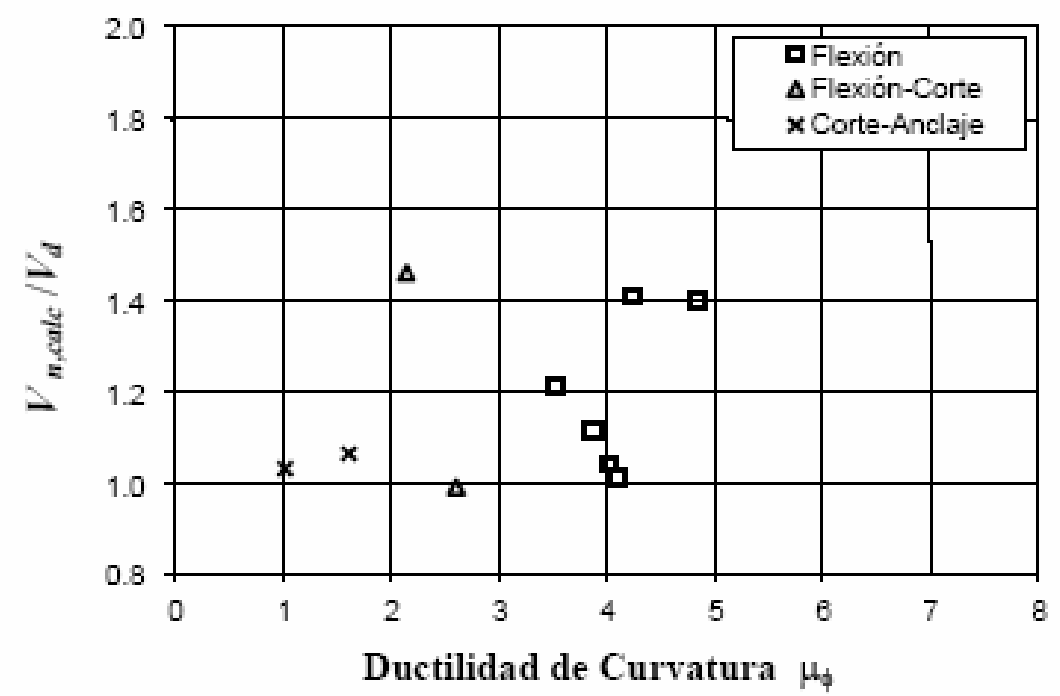

Figura 13 Cuociente de resistencias calculada con modelo propuesto y experimental, para muros que no fallan por corte

\section{CONCLUSIONES}

El análisis de los datos experimentales disponibles en la literatura ha permitido detectar claramente dos importantes efectos. En primer lugar, se determinó que la contribución del refuerzo transversal a la resistencia al corte de muros de hormigón armado alcanza solamente a una fracción del valor entregado por las normas de diseño. Para los datos analizados, esta fracción es aproximadamente un 70\%. En segundo lugar, se detectó claramente el efecto de degradación de la contribución del hormigón a la resistencia al corte, originada por la demanda de deformaciones no lineales en flexión y el consecuente daño asociado.

A partir de un modelo teórico basado en el estudio analítico del mecanismo de transferencia de corte a través de la cabeza de compresión del muro, y del análisis de los resultados experimentales, se ha desarrollado un modelo de resistencia al corte que incluye los efectos antes mencionados. Dados los datos experimentales disponibles, la demanda de deformaciones no lineales en flexión fue evaluada mediante la ductilidad de curvatura alcanzada al instante de la falla.

Los resultados de este estudio, donde se proponen cambios importantes en la forma tradicional de calcular la resistencia al corte, deben ser considerados como un primer intento de resolver el problema. La evaluación del daño sufrido por los elementos estructurales y su efecto sobre la pérdida de resistencia, debe realizarse considerando no sólo las deformaciones alcanzadas, sino la historia total de estas deformaciones. Es necesario además contar con 
resultados experimentales que permitan verificar algunas de las hipótesis aquí enunciadas, identificar y estudiar los diferentes mecanismos de falla al corte, tales como tensión diagonal o compresión diagonal, y aclarar las definiciones de resistencia y estado último de los elementos. Resultados posteriores obtenidos por los autores de este trabajo, que serán presentados en breve, han entregado valiosa información al respecto.

Tabla 1 Valores Estadísticos de las distribuciones de los Cuocientes entre la Resistencia al Corte Calculada y la Experimental

\begin{tabular}{|l|c|c|c|c|}
\hline \multicolumn{1}{|c|}{ Modelo Analítico } & $\begin{array}{c}\text { Valor } \\
\text { medio }\end{array}$ & $\begin{array}{c}\text { Coeficiente de } \\
\text { Variación \% }\end{array}$ & $\mathbf{P}_{\mathbf{9 0}}$ & $\mathbf{P}_{\mathbf{1 0}}$ \\
\hline ACI318-95 Cap. 11, ACI, 1995 & 0.81 & 23.4 & 0.57 & 1.05 \\
\hline ACI318-95 Cap. 21, ACI, 1995 & 0.97 & 23.7 & 0.68 & 1.26 \\
\hline Barda et.al., 1997 & 1.11 & 19.8 & 0.83 & 1.39 \\
\hline Hirosawa et.al., 1988 & 1.04 & 15.4 & 0.84 & 1.24 \\
\hline Diseño AIJ Japon, AIJ, 1988 & 1.60 & 20.6 & 1.18 & 2.02 \\
\hline Hernández y Zermeño, 1980 & 1.06 & 24.5 & 0.73 & 1.39 \\
\hline
\end{tabular}

\section{RECONOCIMIENTOS}

Este trabajo fue realizado durante el desarrollo del Proyecto de Investigación №1950391 del Fondo de Ciencia y Tecnología de Chile (FONDECYT), institución que financió las investigaciones.

\section{REFERENCIAS}

ACI Committee 318 (1995), Building Code Requirements for Structural Concrete (ACI 318-95) and Commentary (318R-95), American Concrete Institute, Farmington Hills, Michigan.

AIJ Structural Committee (1988), Design for Earthquake Resistant Reinforced Concrete Buildings Based on Ultimate Strength Concept, Architectural Institute of Japan.

ATC (1981), Seismic Design Guidelines for Highway Bridges, ATC-6, Applied Technology Council, Berkeley. 
Barda, F.; Hanson, J.M.; and Corley, W.G. (1977), "Shear Strength of Low-Rise Walls with Boundary Elements," Reinforced Concrete Structures in Seismic Zones, Publication SP53, American Concrete Institute, pp. 149-203.

Cardenas, A.E.; Hanson, J.M.; Corley, G.; and Hognestad, E. (1973), "Design Provisions for Shear Walls," ACI Structural Journal, V.70, No.3, pp. 221-230.

Hernández, O. and Zermeño, M. (1980), "Strength and Behavior of Structural Walls with Shear Failure," Proceedings, Structural Aspects Part I, Seventh World Conference on Earthquake Engineering, Istanbul, Turkey, pp.121-124.

Hidalgo, P. A. and Jordan, R. M. (1996), "Strenght and Energy Dissipation Characteristics of Reinforced Concrete Walls under Shear Failure", Proceedings of The Eleventh World Conference on Earthquake Engineering, Acapulco, Mexico.

Hirosawa, M., Hiraishi, H., Fujisawa, M., and Yoshimura, M. (1988), "Seismic Performance of Reinforced Concrete High-Rise Frame Structures with Wall Columns - Part 1: Deformation Capacity of Critical Structural Elements such as Wall Columns, Beams, and Shear Walls", Proceedings, $20^{\text {th }}$ Joint Meeting US-Japan Panel on Wind and Seismic Effects, Washington D.C., U.S.A.

Jordan, R. y Lüders, C. (1993), "Resistencia al Corte de Muros de Hormigón Armado", Anales, Sextas Jornadas Chilenas de Sismología e Ingeniería Antisísmica, Vol.1, Santiago, Chile, pp.621-629.

Kou, A.E. (1995), Un Modelo Histerético para Muros de Hormigón Armado que Fallan por Esfuerzos de Corte, Tesis para optar el grado de Magister en Ciencias de la Ingeniería, Pontificia Universidad Católica de Chile, Santiago de Chile.

Larenas, J.L. (1994), Comportamiento Sísmico de Muros de Hormigón Armado que Fallan por Esfuerzos de Corte, Tesis para optar al grado de Magister en Ciencias de la Ingeniería, Pontificia Universidad Católica de Chile, Santiago de Chile.

Pilakoutas, K.; and Elnashai, A.S. (1995), "Cyclic Behavior of Reinforced Concrete Cantilever Walls, Part I: Experimental Results,” ACI Structural Journal, V.92, No.3, pp. 271-281.

Pilakoutas, K. and Elnashai, A.S. (1995), "Cyclic Behavior of Reinforced Concrete Cantilever Walls, Part II: Discussions and Theoretical Comparisons,” ACI Structural Journal, V.92, No.4, pp. 425-434.

Wood, Sharon L. (1989), "Minimum Tensile Reinforcement Requirements in Walls," $A C I$ Structural Journal, V.86, No.4, pp.582-591.

Wood, Sharon L. (1990), "Shear Strength of Low-Rise reinforced Concrete Walls," ACI Structural Journal, V.87, No.1, pp.99-107. 